\title{
Article \\ The Equivalent Conditions of the Optimal Hilbert-Type Multiple Series Inequality with Quasi-Homogeneous Kernel
}

\author{
Yong Hong ${ }^{1}$, Liju Lu ${ }^{2}$ and Bing $\mathrm{He}^{3, *}$
}

check for

updates

Citation: Hong, Y.; Lu, L.; He, B. The Equivalent Conditions of the Optimal Hilbert-Type Multiple Series Inequality with Quasi-Homogeneous Kernel. Symmetry 2022, 14, 520.

https://doi.org/10.3390/

sym 14030520

Academic Editor: Mihai Postolache

Received: 9 February 2022

Accepted: 28 February 2022

Published: 3 March 2022

Publisher's Note: MDPI stays neutral with regard to jurisdictional claims in published maps and institutional affiliations.

Copyright: () 2022 by the authors Licensee MDPI, Basel, Switzerland. This article is an open access article distributed under the terms and conditions of the Creative Commons Attribution (CC BY) license (https:// creativecommons.org/licenses/by/ $4.0 /)$.
1 Department of Applied Mathematics, Guangzhou Huashang College, Guangzhou 511300, China; hongyong@gdufe.edu.cn

2 Department of Foundation Courses, Guangdong Polytechnic of Environmental Protection Engineering, Guangzhou 511300, China; luliju020@163.com

3 Department of Mathematics, Guangdong University of Education, Guangzhou 510303, China

* Correspondence: hebing@gdei.edu.cn

\begin{abstract}
By introducing several independent parameters, according to the structural symmetry of quasi-homogeneous kernels and the Hilbert-type inequality, and using the weight function method, the parameter conditions of the optimal Hilbert-type $n$-multiple series inequality with quasi-homogeneous kernels are discussed, and several equivalent conditions and the expression formula of the best constant factor are obtained. As applications, some special symmetric inequalities are given.
\end{abstract}

Keywords: quasi-homogeneous kernel; Hilbert-type multiple series inequality; the best constant factor; equivalent condition

JEL Classification: 26D15; 47A07

\section{Introduction}

Suppose that $\tilde{a}=\left\{a_{m}\right\} \in l_{2}, \tilde{b}=\left\{b_{n}\right\} \in l_{2}$. In 1908, the literature [1] stated the well-known Hilbert series inequality with symmetric and -1 order homogeneous kernel $\frac{1}{m+n}:$

$$
\sum_{n=1}^{\infty} \sum_{m=1}^{\infty} \frac{1}{m+n} a_{m} b_{n} \leq \pi\left(\sum_{m=1}^{\infty} a_{m}^{2}\right)^{1 / 2}\left(\sum_{n=1}^{\infty} b_{n}^{2}\right)^{1 / 2}=\pi\|\tilde{a}\|_{2}\|\tilde{b}\|_{2},
$$

where the constant factor $\pi$ is the best. In 1925, by introducing a pair of conjugate parameters $(p, q)\left(\frac{1}{p}+\frac{1}{q}=1, p>1\right)$, Hardy [2] generalized (1) as follows: If $\tilde{a}=\left\{a_{m}\right\} \in l_{p}$, $\tilde{b}=\left\{b_{n}\right\} \in l_{q}$, then

$$
\begin{aligned}
\sum_{n=1}^{\infty} \sum_{m=1}^{\infty} \frac{1}{m+n} a_{m} b_{n} & \leq \frac{\pi}{\sin (\pi / p)}\left(\sum_{m=1}^{\infty}\left|a_{m}\right|^{p}\right)^{1 / p}\left(\sum_{n=1}^{\infty}\left|b_{n}\right|^{q}\right)^{1 / q} \\
& =\frac{\pi}{\sin (\pi / p)}\|\tilde{a}\|_{p}\|\tilde{b}\|_{q},
\end{aligned}
$$

where the constant factor $\pi / \sin (\pi / p)$ is the best.

Define the series operator $T$ by

$$
T(\tilde{a})_{n}=\sum_{m=1}^{\infty} \frac{1}{m+n} a_{m}, \tilde{a}=\left\{a_{m}\right\} \in l_{p}, n=1,2, \cdots
$$


then, we can prove that (2) has the following dual operator representation:

$$
\|T(\tilde{a})\|_{p} \leq \frac{\pi}{\sin (\pi / p)}\|\tilde{a}\|_{p} .
$$

It follows that $T$ is a bounded operator on $l_{p}$, and the operator norm of $T$ is $\|T\|=$ $\pi / \sin (\pi / p)$. Therefore, it has important applications to study (2).

By introducing an independent parameter $\lambda$, (2) has been extended to more general forms [3-5]. In 2020, ref. [6] considered the symmetric homogeneous kernel $(\min \{m, n\})^{\lambda}$ of $\lambda$-order, and obtained the Hilbert-type series inequality of the following form: If $\widetilde{\lambda}_{1}+$ $\widetilde{\lambda}_{2}=\lambda, a_{m} \geq 0, b_{m} \geq 0$, then

$$
\sum_{n=1}^{\infty} \sum_{m=1}^{\infty}(\min \{m, n\})^{\lambda} a_{m} b_{n} \leq M\left(\sum_{m=1}^{\infty} m^{p\left(1+\tilde{\lambda}_{1}\right)-1} a_{m}^{p}\right)^{1 / p}\left(\sum_{n=1}^{\infty} n^{q\left(1+\widetilde{\lambda}_{2}\right)-1} b_{n}^{q}\right)^{1 / q}
$$

and the equivalent conditions and expression formula for the best constant factor are discussed. In particular, when $\widetilde{\lambda}_{1}+\widetilde{\lambda}_{2}=1$, the inequality with the best constant factor 2 is given:

$$
\sum_{n=1}^{\infty} \sum_{m=1}^{\infty}(\min \{m, n\})^{2} a_{m} b_{n} \leq 2\left(\sum_{m=1}^{\infty} m^{2 p-1} a_{m}^{p}\right)^{1 / p}\left(\sum_{n=1}^{\infty} n^{2 q-1} b_{n}^{q}\right)^{1 / q} .
$$

The obvious feature of the above results is that their kernels are symmetric and homogeneous, and the structures of the inequalities also have some symmetry. For further study, we first generalize the space $l_{p}$ to a weighted sequence space:

$$
l_{p}^{\alpha}:=\left\{\tilde{a}=\left\{a_{m}\right\}:\|\tilde{a}\|_{p, \alpha}=\left(\sum_{m=1}^{\infty} m^{\alpha}\left|a_{m}\right|^{p}\right)^{1 / p}<+\infty\right\} .
$$

Next, on the basis of a symmetric homogeneous kernel, we will consider the quasihomogeneous kernel $K(m, n)=G\left(m^{\lambda_{1}}, n^{\lambda_{2}}\right)$, where $G$ is a homogeneous function of $\lambda$-order. For $\tilde{a}=\left\{a_{m}\right\} \in l_{p}^{\alpha}, \tilde{b}=\left\{b_{n}\right\} \in l_{q}^{\beta}, K(m, n) \geq 0$, we call

$$
\sum_{n=1}^{\infty} \sum_{m=1}^{\infty} K(m, n) a_{m} b_{n} \leq M \mid\|\tilde{a}\|_{p, \alpha}\|\tilde{b}\|_{q, \beta}
$$

the Hilbert-type series inequality. For $n \geq 2, \sum_{i=1}^{n} \frac{1}{p_{i}}=1\left(p_{i}>1\right), K\left(m_{1}, m_{2}, \cdots, m_{n}\right) \geq 0$, $\tilde{a}(i)=\left\{a_{m_{i}}(i)\right\} \in l_{p_{i}}^{\alpha_{i}}(i=1,2, \cdots, n)$, we say that

$$
\sum_{m_{1}=1}^{\infty} \sum_{m_{2}=1}^{\infty} \cdots \sum_{m_{n}=1}^{\infty} K\left(m_{1}, m_{2}, \cdots, m_{n}\right) \prod_{i=1}^{n} a_{m_{i}}(i) \leq M \prod_{i=1}^{n}\|\tilde{a}(i)\|_{p_{i}, \alpha_{i}}
$$

is the Hilbert-type $n$-multiple series inequality.

Several results have been obtained on the $n$-multiple Hilbert-type inequality [7-11]. However, the equivalent conditions and the expression formula of the best constant factor for quasi-homogeneous kernel have not yet been seen in the literature. The purpose of this paper is to solve this problem. 
By introducing matching parameters $a_{1}, a_{2}, \cdots, a_{n}$, and using the Hölder's inequality and weight coefficient method, we can obtain the following Hilbert-type multiple series inequality

$$
\begin{aligned}
& \sum_{m_{1}=1}^{\infty} \sum_{m_{2}=1}^{\infty} \cdots \sum_{m_{n}=1}^{\infty} K\left(m_{1}, m_{2}, \cdots, m_{n}\right) \prod_{i=1}^{n} a_{m_{i}}(i) \\
\leq & M\left(p_{1}, \cdots, p_{n}, a_{1}, \cdots, a_{n}\right) \prod_{i=1}^{n}\|\tilde{a}(i)\|_{p_{i}, \alpha_{i}\left(p_{1}, \cdots, p_{n}, a_{1}, \cdots, a_{n}\right)}
\end{aligned}
$$

where the constant factor $M$ and parameters $\left\{\alpha_{i}\right\}_{i=1}^{n}$ all depend on $p_{1}, \cdots, p_{n}$ and $a_{1}, \cdots, a_{n}$. Generally speaking, for any matching parameters $a_{1}, \cdots, a_{n}$, the constant factor $M\left(p_{1}, \cdots\right.$, $\left.p_{n}, a_{1}, \cdots, a_{n}\right)$ in (4) is not the optimal value. To optimize the constant factor in (4), the matching parameters should satisfy certain conditions. In this paper, we discuss the law of best matching parameters in (4), obtain several equivalent conditions of the best matching parameters, and solve the theoretical problem of optimal matching parameters (see Theorem 1 in Section 3).

It is worth pointing out that the structural symmetry of the Hilbert-type inequality makes our treatment of each variable $m_{i}$ of universal significance, which is very important.

\section{Preliminary Lemmas}

Lemma 1. ([12]) Assume that $p_{i}>0, a_{i}>0, \alpha_{i}>0(i=1,2, \cdots, n), \psi(u)$ is measurable. Then

$$
\begin{aligned}
& \int_{\sum_{i=1}^{n}\left(\frac{x_{i}}{a_{i}}\right)^{\alpha_{i}} \leq 1, x_{i}>0} \psi\left(\sum_{i=1}^{n}\left(\frac{x_{i}}{a_{i}}\right)^{\alpha_{i}}\right) \prod_{i=1}^{n} x_{i}^{p_{i}-1} \mathrm{~d} x_{1} \cdots \mathrm{d} x_{n} \\
= & \frac{a_{1}^{p_{1}} \cdots a_{n}^{p_{n}} \Gamma\left(\frac{p_{1}}{\alpha_{1}}\right) \cdots \Gamma\left(\frac{p_{n}}{\alpha_{n}}\right)}{\alpha_{1} \cdots \alpha_{n} \Gamma\left(\frac{p_{1}}{\alpha_{1}}+\cdots+\frac{p_{n}}{\alpha_{n}}\right)} \int_{0}^{1} \psi(u) u^{\sum_{i=1}^{n} \frac{p_{i}}{\alpha_{i}}-1} \mathrm{~d} u,
\end{aligned}
$$

where $\Gamma(t)$ is the Gamma function.

By using Lemma 1, it is not difficult to obtain the following formula.

Lemma 2. Under the conditions of Lemma 1, we have

$$
\begin{aligned}
& \int_{\mathbb{R}_{+}^{n}} \psi\left(\sum_{i=1}^{n}\left(\frac{x_{i}}{a_{i}}\right)^{\alpha_{i}}\right) \prod_{i=1}^{n} x_{i}^{p_{i}-1} \mathrm{~d} x_{1} \cdots \mathrm{d} x_{n} \\
= & \frac{a_{1}^{p_{1}} \cdots a_{n}^{p_{n}} \Gamma\left(\frac{p_{1}}{\alpha_{1}}\right) \cdots \Gamma\left(\frac{p_{n}}{\alpha_{n}}\right)}{\alpha_{1} \cdots \alpha_{n} \Gamma\left(\frac{p_{1}}{\alpha_{1}}+\cdots+\frac{p_{n}}{\alpha_{n}}\right)} \int_{0}^{+\infty} \psi(u) u^{\sum_{i=1}^{n} \frac{p_{i}}{\alpha_{i}}-1} \mathrm{~d} u,
\end{aligned}
$$

where $\mathbb{R}_{+}^{n}=\left\{\left(x_{1}, \cdots, x_{n}\right) \in \mathbb{R}^{n}: x_{i}>0(i=1,2, \cdots, n)\right\}$.

Lemma 3. Suppose that $n \geq 2, \sum_{i=1}^{n} \frac{1}{p_{i}}=1\left(p_{i}>1\right), \lambda_{i} \lambda_{j}>0(i, j=1,2, \cdots, n)$, $K\left(x_{1}, x_{2}, \cdots, x_{n}\right)=G\left(x_{1}^{\lambda_{1}}, x_{2}^{\lambda_{2}}, \cdots, x_{n}^{\lambda_{n}}\right), G\left(u_{1}, u_{2}, \cdots, u_{n}\right)$ is a homogeneous non-negative measurable function of order $\lambda, K\left(x_{1}, \cdots, x_{i}, \cdots, x_{n}\right) x_{i}^{-a_{i}}$ is monotonically decreasing with respect to $x_{i}$ on $(0,+\infty)$, denote

$$
W_{j}=\int_{\mathbb{R}_{+}^{n-1}} K\left(t_{1}, \cdots, t_{j-1}, 1, t_{j+1} \cdots, t_{n}\right) \prod_{i \neq j}^{n} t_{i}^{-a_{i}} \mathrm{~d} t_{1} \cdots \mathrm{d} t_{j-1} \mathrm{~d} t_{j+1} \cdots \mathrm{d} t_{n}
$$


Then

$$
\begin{aligned}
\omega_{j}\left(m_{j}\right) & =\sum_{m_{1}=1}^{\infty} \cdots \sum_{m_{j-1}=1}^{\infty} \sum_{m_{j+1}=1}^{\infty} \cdots \sum_{m_{n}=1}^{\infty} K\left(m_{1}, m_{2}, \cdots, m_{n}\right) \prod_{i \neq j}^{n} m_{i}^{-a_{i}} \\
& \leq m_{j}^{\lambda_{j}\left(\lambda-\sum_{i \neq j}^{n} \frac{a_{i}}{\lambda_{i}}+\sum_{i \neq j}^{n} \frac{1}{\lambda_{i}}\right)} W_{j} .
\end{aligned}
$$

For $\sum_{i=1}^{n} \frac{a_{i}}{\lambda_{i}}=\lambda+\sum_{i=1}^{n} \frac{1}{\lambda_{i}}$, we have $\frac{1}{\lambda_{1}} W_{1}=\frac{1}{\lambda_{2}} W_{2}=\cdots=\frac{1}{\lambda_{n}} W_{n}$.

Proof. First, notice that the quasi-homogeneous kernel $K\left(m_{1}, m_{2}, \cdots, m_{n}\right)$ has an obvious property: For $t>0$,

$$
\begin{aligned}
& K\left(m_{1}, \cdots, m_{j-1}, t m_{j}, m_{j+1} \cdots, m_{n}\right) \\
= & t^{\lambda \lambda_{j}} K\left(t^{-\lambda_{j} / \lambda_{1}} m_{1}, \cdots, t^{-\lambda_{j} / \lambda_{j-1}} m_{j-1}, m_{j}, t^{-\lambda_{j} / \lambda_{j+1}} m_{j+1}, \cdots, t^{-\lambda_{j} / \lambda_{n}} m_{n}\right) .
\end{aligned}
$$
then

Since $K\left(x_{1}, \cdots, x_{i}, \cdots, x_{n}\right) x_{i}^{-a_{i}}$ is monotonically decreasing with respect to $x_{i}$ on $(0,+\infty)$,

$$
\begin{aligned}
& \omega_{1}\left(m_{1}\right)=\sum_{m_{2}=1}^{\infty} \cdots \sum_{m_{n}=1}^{\infty} K\left(m_{1}, m_{2}, \cdots, m_{n}\right) \prod_{i=2}^{n} m_{i}^{-a_{i}} \\
& \leq \sum_{m_{2}=1}^{\infty} \cdots \sum_{m_{n-1}=1}^{\infty} \prod_{i=2}^{n-1} m_{i}^{-a_{i}} \int_{0}^{+\infty} K\left(m_{1}, m_{2}, \cdots, m_{n-1}, t_{n}\right) t_{n}^{-a_{n}} \mathrm{~d} t_{n} \\
& \leq \sum_{m_{2}=1}^{\infty} \cdots \sum_{m_{n-2}=1}^{\infty} \prod_{i=2}^{n-2} m_{i}^{-a_{i}} \int_{\mathbb{R}_{+}^{2}} K\left(m_{1}, m_{2}, \cdots, m_{n-2}, t_{n-1}, t_{n}\right) \prod_{i=n-1}^{n} t_{i}^{-a_{i}} \mathrm{~d} t_{n-1} \mathrm{~d} t_{n} \\
& \leq \ldots \ldots \\
& \leq \int_{\mathbb{R}_{+}^{n-1}} K\left(m_{1}, t_{2}, \cdots, t_{n}\right) \prod_{i=2}^{n} t_{i}^{-a_{i}} \mathrm{~d} t_{2} \cdots \mathrm{d} t_{n} \\
& =m_{1}^{\lambda \lambda_{1}} \int_{\mathbb{R}_{+}^{n-1}} K\left(1, m_{1}^{-\lambda_{1} / \lambda_{2}} t_{2}, \cdots, m_{1}^{-\lambda_{1} / \lambda_{n}} t_{n}\right) \prod_{i=2}^{n} t_{i}^{-a_{i}} \mathrm{~d} t_{2} \cdots \mathrm{d} t_{n} \\
& =m_{1}^{\lambda_{1}\left(\lambda-\sum_{i=2}^{n} \frac{a_{i}}{\lambda_{i}}+\sum_{i=2}^{n} \frac{1}{\lambda_{i}}\right)} \int_{\mathbb{R}_{+}^{n-1}} K\left(1, u_{2}, \cdots, u_{n}\right) \prod_{i=2}^{n} u_{i}^{-a_{i}} \mathrm{~d} u_{2} \cdots \mathrm{d} u_{n} \\
& =m_{1}^{\lambda_{1}\left(\lambda-\sum_{i=2}^{n} \frac{a_{i}}{\lambda_{i}}+\sum_{i=2}^{n} \frac{1}{\lambda_{i}}\right)} W_{1} \text {. }
\end{aligned}
$$

Similarly, we can prove the cases of $j=2,3, \cdots, n$.

For $\sum_{i=1}^{n} \frac{a_{i}}{\lambda_{i}}=\lambda+\sum_{i=1}^{n} \frac{1}{\lambda_{i}}, j=2,3, \cdots, n$, we compute

$$
\begin{aligned}
W_{j}= & \int_{\mathbb{R}_{+}^{n-1}} t^{\lambda \lambda_{1}} K\left(1, t_{1}^{-\lambda_{1} / \lambda_{2}} t_{2}, \cdots, t_{1}^{-\lambda_{1} / \lambda_{j-1}} t_{j-1}, t_{1}^{-\lambda_{1} / \lambda_{j}}, t_{1}^{-\lambda_{1} / \lambda_{j+1}} t_{j+1}, \cdots, t_{1}^{-\lambda_{1} / \lambda_{n}} t_{n}\right) \\
& \times \prod_{i \neq j}^{n} t_{i}^{-a_{i}} \mathrm{~d} t_{1} \cdots \mathrm{d} t_{j-1} \mathrm{~d} t_{j+1} \cdots \mathrm{d} t_{n} \\
= & \frac{\lambda_{j}}{\lambda_{1}} \int_{\mathbb{R}_{+}^{n-1}} K\left(1, u_{2}, \cdots, u_{n}\right) u_{j}^{\lambda_{j}\left(\sum_{i=1}^{n} \frac{a_{i}}{\lambda_{i}}-\lambda-\sum_{i=1}^{n} \frac{1}{\lambda_{i}}\right)-a_{j}} \prod_{i \neq 1, i \neq j}^{n} u_{i}^{-a_{i}} \mathrm{~d} u_{2} \cdots \mathrm{d} u_{n} \\
= & \frac{\lambda_{j}}{\lambda_{1}} \int_{\mathbb{R}_{+}^{n-1}} K\left(1, u_{2}, \cdots, u_{n}\right) \prod_{i=2}^{n} u_{i}^{-a_{i}} \mathrm{~d} u_{2} \cdots \mathrm{d} u_{n}=\frac{\lambda_{j}}{\lambda_{1}} W_{1} .
\end{aligned}
$$

Hence $\frac{1}{\lambda_{1}} W_{1}=\frac{1}{\lambda_{j}} W_{j}(j=2,3, \cdots, n)$. 


\section{Main Results}

In this section, we introduce the matching parameters $a_{1}, a_{2}, \cdots, a_{n}$, and use the weight coefficient method to establish the Hilbert inequality of $n$-multiple series in Theorem 1(i), and obtain two equivalent conditions of the best matching parameters in Theorem 1(ii).

Theorem 1. Suppose that $n \geq 2, \sum_{i=1}^{n} \frac{1}{p_{i}}=1\left(p_{i}>1\right), a_{i} \in \mathbb{R}, \lambda_{i} \lambda_{j}>0(i, j=1,2, \cdots, n)$, $K\left(x_{1}, x_{2}, \cdots, x_{n}\right)=G\left(x_{1}^{\lambda_{1}}, x_{2}^{\lambda_{2}}, \cdots, x_{n}^{\lambda_{n}}\right)>0, G\left(u_{1}, u_{2}, \cdots, u_{n}\right)$ is a homogeneous non-negative measurable function of order $\lambda, \sum_{i=1}^{n} \frac{a_{i}}{\lambda_{i}}-\left(\lambda+\sum_{i=1}^{n} \frac{1}{\lambda_{i}}\right)=c, K\left(x_{1}, \cdots, x_{i}, \cdots, x_{n}\right) x_{i}^{-a_{i}}$ and $K\left(x_{1}, \cdots, x_{i}, \cdots, x_{n}\right) x_{i}^{-a_{i}+\frac{\lambda_{i} c}{p_{i}}}$ are monotonically decreasing with respect to $x_{i}$ on $(0,+\infty)$, and for $j=1,2, \cdots, n$,

$$
W_{j}=\int_{\mathbb{R}_{+}^{n-1}} G\left(t_{1}^{\lambda_{1}}, \cdots, t_{j-1}^{\lambda_{j-1}}, 1, t_{j+1}^{\lambda_{j+1}}, \cdots, t_{n}^{\lambda_{n}}\right) \prod_{i \neq j}^{n} t_{i}^{-a_{i}} \mathrm{~d} t_{1} \cdots \mathrm{d} t_{j-1} \mathrm{~d} t_{j+1} \cdots \mathrm{d} t_{n}
$$

is convergent, then:

(i) Denote

$$
\alpha_{i}=\lambda_{i}\left(\lambda+\sum_{k \neq i}^{n} \frac{1}{\lambda_{k}}-\sum_{k=1}^{n} \frac{a_{k}}{\lambda_{k}}\right)+a_{i} p_{i}
$$

one has

$$
\sum_{m_{1}=1}^{\infty} \cdots \sum_{m_{n}=1}^{\infty} G\left(m_{1}^{\lambda_{1}}, m_{2}^{\lambda_{2}}, \cdots, m_{n}^{\lambda_{n}}\right) \prod_{i=1}^{n} a_{m_{i}}(i) \leq\left.\left(\prod_{i=1}^{n} W_{i}^{1 / p_{i}}\right) \prod_{i=1}^{n}\|\tilde{a}(i)\|\right|_{p_{i}, \alpha_{i}},
$$

where $\tilde{a}(i)=\left\{a_{m_{i}}(i)\right\} \in l_{p_{i}}^{\alpha_{i}}(i=1,2, \cdots, n)$.

(ii) The following three conditions are equivalent:

(a) The constant factor $\prod_{i=1}^{n} W_{i}^{1 / p_{i}}$ of (5) is the best. That is, $a_{1}, a_{2}, \cdots, a_{n}$ are the best matching parameters;

(b) $\sum_{i=1}^{n} \frac{a_{i}}{\lambda_{i}}=\lambda+\sum_{i=1}^{n} \frac{1}{\lambda_{i}}$;

(c) $\frac{1}{\lambda_{1}} W_{1}=\frac{1}{\lambda_{2}} W_{2}=\cdots=\frac{1}{\lambda_{n}} W_{n}$.

(iii) For $\sum_{i=1}^{n} \frac{a_{i}}{\lambda_{i}}=\lambda+\sum_{i=1}^{n} \frac{1}{\lambda_{i}}$, (5) becomes

$$
\sum_{m_{1}=1}^{\infty} \cdots \sum_{m_{n}=1}^{\infty} G\left(m_{1}^{\lambda_{1}}, m_{2}^{\lambda_{2}}, \cdots, m_{n}^{\lambda_{n}}\right) \prod_{i=1}^{n} a_{m_{i}}(i) \leq\left(W_{0} \prod_{i=1}^{n}\left|\lambda_{i}\right|^{1 / p_{i}}\right) \prod_{i=1}^{n}\|\tilde{a}(i)\|_{p_{i}, a_{i} p_{i-1}},
$$

where $W_{0}=\frac{1}{\left|\lambda_{1}\right|} W_{1}=\frac{1}{\left|\lambda_{2}\right|} W_{2}=\cdots=\frac{1}{\left|\lambda_{n}\right|} W_{n}$.

Proof. (i) Since

$$
\prod_{j=1}^{n}\left[m_{j}^{a_{j}}\left(\prod_{i=1}^{n} m_{i}^{-a_{i}}\right)^{1 / p_{j}}\right]=\left(\prod_{i=1}^{n} m_{i}^{-a_{i}}\right)^{\frac{1}{p_{1}}+\cdots+\frac{1}{p_{n}}} \prod_{j=1}^{n} m_{j}^{a_{j}}=\prod_{i=1}^{n} m_{i}^{-a_{i}} \prod_{j=1}^{n} m_{j}^{a_{j}}=1,
$$


it follows from Hölder's inequality and Lemma 3 that

$$
\begin{aligned}
& \sum_{m_{1}=1}^{\infty} \cdots \sum_{m_{n}=1}^{\infty} G\left(m_{1}^{\lambda_{1}}, m_{2}^{\lambda_{2}}, \cdots, m_{n}^{\lambda_{n}}\right) \prod_{i=1}^{n} a_{m_{i}}(i) \\
& =\sum_{m_{1}=1}^{\infty} \cdots \sum_{m_{n}=1}^{\infty} K\left(m_{1}, m_{2}, \cdots, m_{n}\right) \prod_{j=1}^{n}\left[m_{j}^{a_{j}} a_{m_{j}}(j)\left(\prod_{i=1}^{n} m_{i}^{-a_{i}}\right)^{1 / p_{j}}\right] \\
& \leq \prod_{j=1}^{n}\left(\sum_{m_{j}=1}^{\infty} m_{j}^{a_{j} p_{j}-a_{j}}\left|a_{m_{j}}(j)\right|^{p_{j}} \omega_{j}\left(m_{i}\right)\right)^{1 / p_{j}} \\
& \leq \prod_{j=1}^{n} W_{j}^{1 / p_{j}} \prod_{j=1}^{n}\left(\sum_{m_{j}=1}^{\infty} m_{j}^{a_{j} p_{j}-a_{j}+\lambda_{j}\left(\lambda-\sum_{i \neq j}^{n} \frac{a_{i}}{\lambda_{i}}+\sum_{i \neq j}^{n} \frac{1}{\lambda_{i}}\right)}\left|a_{m_{j}}(j)\right|^{p_{j}}\right)^{1 / p_{j}} \\
& =\prod_{i=1}^{n} W_{i}^{1 / p_{i}} \prod_{i=1}^{n}\left(\sum_{m_{i}=1}^{\infty} m_{i}^{\alpha_{i}}\left|a_{m_{i}}(i)\right|^{p_{i}}\right)^{1 / p_{i}}=\left(\prod_{i=1}^{n} W_{i}^{1 / p_{i}}\right) \prod_{i=1}^{n}\|\tilde{a}(i)\|_{p_{i}, \alpha_{i}} .
\end{aligned}
$$

Hence, (5) holds.

(ii) $(\mathrm{b}) \Rightarrow$ (c) By Lemma 3 we have (b) $\Rightarrow$ (c).

(b) $\Rightarrow$ (a) Suppose that $\sum_{i=1}^{n} \frac{a_{i}}{\lambda_{i}}=\lambda+\sum_{i=1}^{n} \frac{1}{\lambda_{i}}$, then $\alpha_{i}=a_{i} p_{i}-1(i=1,2, \cdots, n)$. It follows from Lemma 3 that $\frac{1}{\lambda_{1}} W_{1}=\frac{1}{\lambda_{2}} W_{2}=\cdots=\frac{1}{\lambda_{n}} W_{n}$, and

$$
\prod_{i=1}^{n} W_{i}^{1 / p_{i}}=W_{1}^{1 / p_{1}} \prod_{i=2}^{n}\left(\frac{\lambda_{i}}{\lambda_{1}} W_{i}\right)^{1 / p_{i}}=\frac{W_{1}}{\left|\lambda_{1}\right|} \prod_{i=1}^{n}\left|\lambda_{i}\right|^{1 / p_{i}}=W_{0} \prod_{i=1}^{n}\left|\lambda_{i}\right|^{1 / p_{i}}
$$

Therefore, (5) becomes (6).

Let the best constant factor of (6) be $M_{0}$, then $M_{0} \leq W_{0} \prod_{i=1}^{n}\left|\lambda_{i}\right|^{1 / p_{i}}$, and (6) still holds after replacing the constant factor of (6) with $M_{0}$.

For sufficiently small $\varepsilon>0$ and sufficiently large natural number $N$, take

$$
\begin{gathered}
a_{m_{i}}(i)=m_{i}^{\left(-a_{i} p_{i}-\left|\lambda_{i}\right| \varepsilon\right) / p_{i}}, i=2,3, \cdots, n, \\
a_{m_{1}}(1)=\left\{\begin{array}{cc}
0, & m_{1}=1,2, \cdots, N-1, \\
m_{1}^{\left(-a_{1} p_{1}-\left|\lambda_{1}\right| \varepsilon\right) / p_{1}}, & m_{1}=N, N+1, \cdots
\end{array}\right.
\end{gathered}
$$

then

$$
\begin{aligned}
\prod_{i=1}^{n}\|\tilde{a}(i)\| p_{i}, a_{i} p_{i-1} & =\left(\sum_{m_{1}=N}^{\infty} m_{1}^{-1-\left|\lambda_{1}\right| \varepsilon}\right)^{1 / p_{1}} \prod_{i=2}^{n}\left(\sum_{m_{i}=1}^{\infty} m_{i}^{-1-\left|\lambda_{i}\right| \varepsilon}\right)^{1 / p_{i}} \\
& \leq\left(\int_{N-1}^{+\infty} t^{-1-\left|\lambda_{1}\right| \varepsilon} \mathrm{d} t\right)^{1 / p_{1}} \prod_{i=2}^{n}\left(1+\int_{1}^{+\infty} t^{-1-\left|\lambda_{i}\right| \varepsilon} \mathrm{d} t\right)^{1 / p_{i}} \\
& =\frac{1}{\varepsilon}(N-1)^{-\left|\lambda_{1}\right| \varepsilon / p_{1}} \prod_{i=1}^{n}\left|\lambda_{i}\right|^{-1 / p_{i}} \prod_{i=2}^{n}\left(\left|\lambda_{i}\right| \varepsilon+1\right)^{1 / p_{i}}
\end{aligned}
$$

Notice that $K\left(x_{1}, \cdots, x_{i}, \cdots, x_{n}\right) x_{i}^{-a_{i}}$ is monotonically decreasing with respect to $x_{i}$ on $(0,+\infty)$, then 


$$
\begin{aligned}
& \sum_{m_{1}=1}^{\infty} \cdots \sum_{m_{n}=1}^{\infty} G\left(m_{1}^{\lambda_{1}}, m_{2}^{\lambda_{2}}, \cdots, m_{n}^{\lambda_{n}}\right) \prod_{i=1}^{n} a_{m_{i}}(i) \\
& =\sum_{m_{1}=N}^{\infty} m_{1}^{\left(-a_{1} p_{1}-\left|\lambda_{1}\right| \varepsilon\right) / p_{1}} \sum_{m_{2}=1}^{\infty} \cdots \sum_{m_{n}=1}^{\infty} K\left(m_{1}, m_{2}, \cdots, m_{n}\right) \prod_{i=2}^{n} m_{i}^{\left(-a_{i} p_{i}-\left|\lambda_{i}\right| \varepsilon\right) / p_{i}} \\
& \geq \sum_{m_{1}=N}^{\infty} m_{1}^{\left(-a_{1} p_{1}-\left|\lambda_{1}\right| \varepsilon\right) / p_{1}} \sum_{m_{2}=1}^{\infty} \cdots \sum_{m_{n-1}=1}^{\infty} \prod_{i=2}^{n-1} m_{i}^{\left(-a_{i} p_{i}-\left|\lambda_{i}\right| \varepsilon\right) / p_{i}} \\
& \times\left(\int_{1}^{+\infty} K\left(m_{1}, \cdots, m_{n-1}, u_{n}\right) u_{n}^{\left(-a_{n} p_{n}-\left|\lambda_{n}\right| \varepsilon\right) / p_{n}} \mathrm{~d} u_{n}\right) \\
& \geq \sum_{m_{1}=N}^{\infty} m_{1}^{\left(-a_{1} p_{1}-\left|\lambda_{1}\right| \varepsilon\right) / p_{1}} \sum_{m_{2}=1}^{\infty} \cdots \sum_{m_{n-2}=1}^{\infty} \prod_{i=2}^{n-2} m_{i}^{\left(-a_{i} p_{i}-\left|\lambda_{i}\right| \varepsilon\right) / p_{i}} \\
& \times\left(\int_{1}^{+\infty} \int_{1}^{+\infty} K\left(m_{1}, \cdots, m_{n-2}, u_{n-1}, u_{n}\right) \prod_{i=n-1}^{n} u_{i}^{\left(-a_{i} p_{i}-\left|\lambda_{i}\right| \varepsilon\right) / p_{i}} \mathrm{~d} u_{n-1} \mathrm{~d} u_{n}\right) \\
& \geq \cdots \cdots \\
& \geq \sum_{m_{1}=N}^{\infty} m_{1}^{\left(-a_{1} p_{1}-\left|\lambda_{1}\right| \varepsilon\right) / p_{1}}\left(\int_{1}^{+\infty} \cdots \int_{1}^{+\infty} K\left(m_{1}, u_{2}, \cdots, u_{n}\right) \prod_{i=2}^{n} u_{i}^{\left(-a_{i} p_{i}-\left|\lambda_{i}\right| \varepsilon\right) / p_{i}} \mathrm{~d} u_{2} \cdots \mathrm{d} u_{n}\right) \\
& =\sum_{m_{1}=N}^{\infty} m_{1}^{\lambda \lambda_{1}-\frac{a_{1} p_{1}+\left|\lambda_{1}\right| \varepsilon}{p_{1}}}\left(\int_{1}^{+\infty} \cdots \int_{1}^{+\infty} K\left(1, m_{1}^{-\frac{\lambda_{1}}{\lambda_{2}}} u_{2}, \cdots, m_{1}^{-\frac{\lambda_{1}}{\lambda_{n}}} u_{n}\right)\right. \\
& \left.\times \prod_{i=2}^{n} u_{i}^{\left(-a_{i} p_{i}-\left|\lambda_{i}\right| \varepsilon\right) / p_{i}} \mathrm{~d} u_{2} \cdots \mathrm{d} u_{n}\right) \\
& =\sum_{m_{1}=N}^{\infty} m_{1}^{\lambda \lambda_{1}-\frac{a_{1} p_{1}+\left|\lambda_{1}\right| \varepsilon}{p_{1}}-\lambda \sum_{i=2}^{n} \frac{a_{i} p_{i}+\left|\lambda_{i}\right| \varepsilon}{\lambda_{i} p_{i}}+\lambda_{1} \sum_{i=2}^{n} \frac{1}{\lambda_{i}}}\left(\int_{m_{1}^{-\lambda_{1} / \lambda_{2}}}^{+\infty} \cdots \int_{m_{1}^{-\lambda_{1} / \lambda_{n}}}^{+\infty} K\left(1, t_{2}, \cdots, t_{n}\right)\right. \\
& \left.\times \prod_{i=2}^{n} t_{i}^{\left(-a_{i} p_{i}-\left|\lambda_{i}\right| \varepsilon\right) / p_{i}} \mathrm{~d} t_{2} \cdots \mathrm{d} t_{n}\right) \\
& \geq \sum_{m_{1}=N}^{\infty} m_{1}^{-1-\left|\lambda_{1}\right| \varepsilon}\left(\int_{N^{-\lambda_{1} / \lambda_{2}}}^{+\infty} \cdots \int_{N^{-\lambda_{1} / \lambda_{n}}}^{+\infty} K\left(1, t_{2}, \cdots, t_{n}\right) \prod_{i=2}^{n} t_{i}^{\left(-a_{i} p_{i}-\left|\lambda_{i}\right| \varepsilon\right) / p_{i}} \mathrm{~d} t_{2} \cdots \mathrm{d} t_{n}\right) \\
& \geq \int_{N}^{+\infty} t_{1}^{-1-\left|\lambda_{1}\right| \varepsilon} \mathrm{d} t_{1}\left(\int_{N^{-\lambda_{1} / \lambda_{2}}}^{+\infty} \cdots \int_{N^{-\lambda_{1} / \lambda_{n}}}^{+\infty} K\left(1, t_{2}, \cdots, t_{n}\right) \prod_{i=2}^{n} t_{i}^{\left(-a_{i} p_{i}-\left|\lambda_{i}\right| \varepsilon\right) / p_{i}} \mathrm{~d} t_{2} \cdots \mathrm{d} t_{n}\right) \\
& =\frac{1}{\left|\lambda_{1}\right| \varepsilon} N^{-\left|\lambda_{1}\right| \varepsilon}\left(\int_{N^{-\lambda_{1} / \lambda_{2}}}^{+\infty} \cdots \int_{N^{-\lambda_{1} / \lambda_{n}}}^{+\infty} K\left(1, t_{2}, \cdots, t_{n}\right) \prod_{i=2}^{n} t_{i}^{\left(-a_{i} p_{i}-\left|\lambda_{i}\right| \varepsilon\right) / p_{i}} \mathrm{~d} t_{2} \cdots \mathrm{d} t_{n}\right),
\end{aligned}
$$

therefore

$$
\begin{aligned}
& \frac{1}{\left|\lambda_{1}\right|} N^{-\left|\lambda_{1}\right| \varepsilon}\left(\int_{N^{-\lambda_{1} / \lambda_{2}}}^{+\infty} \cdots \int_{N^{-\lambda_{1} / \lambda_{n}}}^{+\infty} K\left(1, t_{2}, \cdots, t_{n}\right) \prod_{i=2}^{n} t_{i}^{\left(-a_{i} p_{i}-\left|\lambda_{i}\right| \varepsilon\right) / p_{i}} \mathrm{~d} t_{2} \cdots \mathrm{d} t_{n}\right) \\
\leq & M_{0}(N-1)^{-\left|\lambda_{1}\right| \varepsilon / p_{1}} \prod_{i=1}^{n}\left|\lambda_{i}\right|^{-1 / p_{i}} \prod_{i=2}^{n}\left(\left|\lambda_{i}\right| \varepsilon+1\right)^{1 / p_{i}} .
\end{aligned}
$$

Letting $\varepsilon \rightarrow 0^{+}$and then $N \rightarrow+\infty$, by calculating the quadratic limit, we obtain

$$
\frac{1}{\left|\lambda_{1}\right|} \int_{\mathbb{R}_{+}^{n-1}} K\left(1, t_{2}, \cdots, t_{n}\right) \prod_{i=2}^{n} t_{i}^{-a_{i}} \mathrm{~d} t_{2} \cdots \mathrm{d} t_{n} \leq M_{0} \prod_{i=1}^{n}\left|\lambda_{i}\right|^{-1 / p_{i}},
$$

consequently,

$$
W_{0} \prod_{i=1}^{n}\left|\lambda_{i}\right|^{1 / p_{i}}=\frac{W_{1}}{\left|\lambda_{1}\right|} \prod_{i=1}^{n}\left|\lambda_{i}\right|^{1 / p_{i}} \leq M_{0}
$$


Thus, the best constant factor of (6) is $M_{0}=W_{0} \prod_{i=1}^{n}\left|\lambda_{i}\right|^{1 / p_{i}}$.

(a) $\Rightarrow$ (b) Let the constant factor $\prod_{i=1}^{n} W_{i}^{1 / p_{i}}$ of (5) be the best. Put $a_{i}^{\prime}=a_{i}-\frac{\lambda_{i} c}{p_{i}}$, then

$$
\sum_{i=1}^{n} \frac{a_{i}^{\prime}}{\lambda_{i}}-\left(\lambda+\sum_{i=1}^{n} \frac{1}{\lambda_{i}}\right)=\sum_{i=1}^{n}\left(\frac{a_{i}}{\lambda_{i}}-\frac{c}{p_{i}}\right)-\left(\lambda+\sum_{i=1}^{n} \frac{1}{\lambda_{i}}\right)=0 .
$$

For $j \geq 2$, it is not difficult to obtain

$$
W_{j}=\frac{\lambda_{j}}{\lambda_{1}} \int_{\mathbb{R}_{+}^{n-1}} G\left(1, t_{2}^{\lambda_{2}}, \cdots, t_{n}^{\lambda_{n}}\right)\left(\prod_{i=2}^{n} t_{i}^{-a_{i}}\right) t_{j}^{-\lambda_{j} c} \mathrm{~d} t_{2} \cdots \mathrm{d} t_{n} .
$$

Additionally, since

$$
\alpha_{i}^{\prime}=\lambda_{i}\left(\lambda+\sum_{k \neq i}^{n} \frac{1}{\lambda_{k}}-\sum_{k=1}^{n} \frac{a_{k}^{\prime}}{\lambda_{k}}\right)+a_{i}^{\prime} p_{i}=\lambda_{i}\left(\lambda+\sum_{k \neq i}^{n} \frac{1}{\lambda_{k}}-\sum_{k=1}^{n} \frac{a_{k}}{\lambda_{k}}\right)+a_{i} p_{i}=\alpha_{i},
$$

then (5) can be written equivalently as

$$
\begin{aligned}
& \sum_{m_{1}=1}^{\infty} \cdots \sum_{m_{n}=1}^{\infty} G\left(m_{1}^{\lambda_{1}}, m_{2}^{\lambda_{2}}, \cdots, m_{n}^{\lambda_{n}}\right) \prod_{i=1}^{n} a_{m_{i}}(i) \\
\leq & W_{1}^{1 / p_{1}} \prod_{j=2}^{n}\left[\frac{\lambda_{j}}{\lambda_{1}} \int_{\mathbb{R}_{+}^{n-1}} G\left(1, t_{2}^{\lambda_{2}}, \cdots, t_{n}^{\lambda_{n}}\right)\left(\prod_{i=2}^{n} t_{i}^{-a_{i}}\right) t_{j}^{\lambda_{j} c} \mathrm{~d} t_{2} \cdots \mathrm{d} t_{n}\right]^{1 / p_{j}} \\
& \times \prod_{i=1}^{n}\|\tilde{a}(i)\|_{p_{i}, a_{i}^{\prime} .}
\end{aligned}
$$

Hence, according to the assumption, the constant factor

$$
\begin{aligned}
& W_{1}^{1 / p_{1}} \prod_{j=2}^{n}\left[\frac{\lambda_{j}}{\lambda_{1}} \int_{\mathbb{R}_{+}^{n-1}} G\left(1, t_{2}^{\lambda_{2}}, \cdots, t_{n}^{\lambda_{n}}\right)\left(\prod_{i=2}^{n} t_{i}^{-a_{i}}\right) t_{j}^{\lambda_{j} c} \mathrm{~d} t_{2} \cdots \mathrm{d} t_{n}\right]^{1 / p_{j}} \\
= & \frac{1}{\left|\lambda_{1}\right|} \prod_{i=1}^{n}\left|\lambda_{i}\right|^{1 / p_{i}} W_{1}^{1 / p_{1}} \prod_{j=2}^{n}\left[\int_{\mathbb{R}_{+}^{n-1}} G\left(1, t_{2}^{\lambda_{2}}, \cdots, t_{n}^{\lambda_{n}}\right)\left(\prod_{i=2}^{n} t_{i}^{-a_{i}}\right) t_{j}^{\lambda_{j} c} \mathrm{~d} t_{2} \cdots \mathrm{d} t_{n}\right]^{1 / p_{j}}
\end{aligned}
$$

of (7) is the best. In view of $\sum_{i=1}^{n} \frac{a_{i}^{\prime}}{\lambda_{i}}-\left(\lambda+\sum_{i=1}^{n} \frac{1}{\lambda_{i}}\right)=0$, and

$$
K\left(x_{1}, \cdots, x_{i}, \cdots, x_{n}\right) x_{i}^{-a_{i}^{\prime}}=K\left(x_{1}, \cdots, x_{i}, \cdots, x_{n}\right) x_{i}^{-a_{i}+\frac{\lambda_{i} c}{p_{i}}}
$$

is monotonically decreasing with respect to $x_{i}$ on $(0,+\infty)$, according to the proof of $(b) \Rightarrow(a)$, we can see that the best constant of (7) is

$$
\begin{aligned}
& \prod_{i=1}^{n}\left|\lambda_{i}\right|^{1 / p_{i}} W_{0}^{\prime}=\prod_{i=1}^{n}\left|\lambda_{i}\right|^{1 / p_{i}}\left(\frac{1}{\left|\lambda_{1}\right|} W_{1}^{\prime}\right) \\
= & \frac{1}{\left|\lambda_{1}\right|} \prod_{i=1}^{n}\left|\lambda_{i}\right|^{1 / p_{i}} \int_{\mathbb{R}_{+}^{n-1}} G\left(1, t_{2}^{\lambda_{2}}, \cdots, t_{n}^{\lambda_{n}}\right) \prod_{i=2}^{n} t_{i}^{-a_{i}^{\prime}} \mathrm{d} t_{2} \cdots \mathrm{d} t_{n} \\
= & \frac{1}{\left|\lambda_{1}\right|} \prod_{i=1}^{n}\left|\lambda_{i}\right|^{1 / p_{i}} \int_{\mathbb{R}_{+}^{n-1}} G\left(1, t_{2}^{\lambda_{2}}, \cdots, t_{n}^{\lambda_{n}}\right)\left(\prod_{i=2}^{n} t_{i}^{-a_{i}}\right) \prod_{i=2}^{n} t_{i}^{\frac{\lambda_{i} c}{p_{i}}} \mathrm{~d} t_{2} \cdots \mathrm{d} t_{n} .
\end{aligned}
$$


Set $H\left(t_{2}, \cdots, t_{n}\right)=G\left(1, t_{2}^{\lambda_{2}}, \cdots, t_{n}^{\lambda_{n}}\right) \prod_{i=2}^{n} t_{i}^{-a_{i}}$, then, in summary, it can be obtained that

$$
\int_{\mathbb{R}_{+}^{n-1}} H\left(t_{2}, \cdots, t_{n}\right) \prod_{i=2}^{n} t_{i}^{\frac{\lambda_{i} c}{p_{i}}} \mathrm{~d} t_{2} \cdots \mathrm{d} t_{n}=W_{1}^{1 / p_{1}} \prod_{j=2}^{n}\left(\int_{\mathbb{R}_{+}^{n-1}} H\left(t_{2}, \cdots, t_{n}\right) t_{j}^{\lambda_{j} c} \mathrm{~d} t_{2} \cdots \mathrm{d} t_{n}\right)^{1 / p_{j}}
$$

For functions $1, t_{2}^{\lambda_{2} c / p_{2}}, \cdots, t_{n}^{\lambda_{n} c / p_{n}}$, it follows from Hölder's inequality that

$$
\begin{aligned}
& \int_{\mathbb{R}_{+}^{n-1}} H\left(t_{2}, \cdots, t_{n}\right) \prod_{i=2}^{n} t_{i}^{\frac{\lambda_{i} c}{p_{i}}} \mathrm{~d} t_{2} \cdots \mathrm{d} t_{n} \\
\leq & \left(\int_{\mathbb{R}_{+}^{n-1}} 1^{p_{1}} H\left(t_{2}, \cdots, t_{n}\right) \mathrm{d} t_{2} \cdots \mathrm{d} t_{n}\right)^{1 / p_{1}} \prod_{j=2}^{n}\left(\int_{\mathbb{R}_{+}^{n-1}} H\left(t_{2}, \cdots, t_{n}\right) t_{j}^{\lambda_{j} c} \mathrm{~d} t_{2} \cdots \mathrm{d} t_{n}\right)^{1 / p_{j}} \\
= & W_{1}^{1 / p_{1}} \prod_{j=2}^{n}\left(\int_{\mathbb{R}_{+}^{n-1}} H\left(t_{2}, \cdots, t_{n}\right) t_{j}^{\lambda_{j c} c} \mathrm{~d} t_{2} \cdots \mathrm{d} t_{n}\right)^{1 / p_{j}} .
\end{aligned}
$$

By (8), we know that (9) takes the equal sign. Then, $t_{j}^{\lambda_{j} c}=$ constant $(j=2,3, \cdots, n)$ is obtained from the condition of equal sign of Hölder's inequality. Thus, $c=0$, i.e., $\sum_{i=1}^{n} \frac{a_{i}}{\lambda_{i}}=\lambda+\sum_{i=1}^{n} \frac{1}{\lambda_{i}}$.

(c) $\Rightarrow$ (b) Assume that $\frac{1}{\lambda_{1}} W_{1}=\frac{1}{\lambda_{2}} W_{2}=\cdots=\frac{1}{\lambda_{n}} W_{n}$. If $\frac{1}{r}+\frac{1}{s}=1(0<r<1, s<0)$, then, by the inverse Hölder's inequality, we find for $j \geq 2$

$$
\begin{aligned}
& \int_{\mathbb{R}_{+}^{n-1}} K\left(t_{1}, \cdots, t_{j-1}, 1, t_{j+1}, \cdots, t_{n}\right) \prod_{i \neq j}^{n} t_{i}^{-a_{i}} \mathrm{~d} t_{1} \cdots \mathrm{d} t_{j-1} \mathrm{~d} t_{j+1} \cdots \mathrm{d} t_{n} \\
& =W_{j}=\frac{\lambda_{j}}{\lambda_{1}} W_{1}=\frac{\lambda_{j}}{\lambda_{1}} \int_{\mathbb{R}_{+}^{n-1}} K\left(1, t_{2}, \cdots, t_{n}\right) \prod_{i=2}^{n} t_{i}^{-a_{i}} \mathrm{~d} t_{2} \cdots \mathrm{d} t_{n} \\
& =\frac{\lambda_{j}}{\lambda_{1}} \int_{\mathbb{R}_{+}^{n-1}} K\left(t_{j}^{-\frac{\lambda_{j}}{\lambda_{1}}}, t_{j}^{-\frac{\lambda_{j}}{\lambda_{2}}} t_{2}, \cdots, t_{j}^{-\frac{\lambda_{j}}{\lambda_{j-1}}} t_{j-1}, 1, t_{j}^{-\frac{\lambda_{j}}{\lambda_{j+1}}} t_{j+1}, \cdots t_{j}^{-\frac{\lambda_{j}}{\lambda_{n}}} t_{n}\right) t_{j}^{\lambda \lambda_{j}} \prod_{i=2}^{n} t_{i}^{-a_{i}} \mathrm{~d} t_{2} \cdots \mathrm{d} t_{n} \\
& =\int_{\mathbb{R}_{+}^{n-1}} K\left(u_{1}, \cdots, u_{j-1}, 1, u_{j+1}, \cdots, u_{n}\right) u_{j}^{-\frac{\lambda_{1}}{\lambda_{j}}\left(\lambda \lambda_{j}-a_{j}\right)-\lambda_{1} \sum_{i=2}^{n} \frac{1}{\lambda_{i}}-1} \\
& \times \prod_{i=2(\neq j)}^{n} u_{j}^{\frac{\lambda_{1}}{\lambda_{i}} a_{i}} u_{i}^{-a_{i}} \mathrm{~d} u_{1} \cdots \mathrm{d} u_{j-1} \mathrm{~d} u_{j+1} \cdots \mathrm{d} u_{n} \\
& =\int_{\mathbb{R}_{+}^{n-1}} K\left(u_{1}, \cdots, u_{j-1}, 1, u_{j+1}, \cdots, u_{n}\right) u_{j}^{\lambda_{1}\left(\sum_{i=2}^{n} \frac{a_{i}}{\lambda_{i}}-\lambda-\sum_{i=1}^{n} \frac{1}{\lambda_{i}}\right)} \prod_{i=2(\neq j)}^{n} u_{i}^{-a_{i}} \\
& \times \mathrm{d} u_{1} \cdots \mathrm{d} u_{j-1} \mathrm{~d} u_{j+1} \cdots \mathrm{d} u_{n} \\
& =\int_{\mathbb{R}_{+}^{n-1}} K\left(u_{1}, \cdots, u_{j-1}, 1, u_{j+1}, \cdots, u_{n}\right) u_{j}^{\lambda_{1}\left(c-\frac{a_{1}}{\lambda_{1}}\right)} \prod_{i=2(\neq j)}^{n} u_{i}^{-a_{i}} \mathrm{~d} u_{1} \cdots \mathrm{d} u_{j-1} \mathrm{~d} u_{j+1} \cdots \mathrm{d} u_{n} \\
& =\int_{\mathbb{R}_{+}^{n-1}} u_{j}^{\lambda_{1} c} K\left(t_{1}, \cdots, t_{j-1}, 1, t_{j+1}, \cdots, t_{n}\right) \prod_{i \neq j}^{n} t_{i}^{-a_{i}} \mathrm{~d} t_{1} \cdots \mathrm{d} t_{j-1} \mathrm{~d} t_{j+1} \cdots \mathrm{d} t_{n} \\
& \geq\left(\int_{\mathbb{R}_{+}^{n-1}} 1^{r} K\left(t_{1}, \cdots, t_{j-1}, 1, t_{j+1}, \cdots, t_{n}\right) \prod_{i \neq j}^{n} t_{i}^{-a_{i}} \mathrm{~d} t_{1} \cdots \mathrm{d} t_{j-1} \mathrm{~d} t_{j+1} \cdots \mathrm{d} t_{n}\right)^{1 / r} \\
& \times\left(\int_{\mathbb{R}_{+}^{n-1}} t_{1}^{\lambda_{1} c s} K\left(t_{1}, \cdots, t_{j-1}, 1, t_{j+1}, \cdots, t_{n}\right) \prod_{i \neq j}^{n} t_{i}^{-a_{i}} \mathrm{~d} t_{1} \cdots \mathrm{d} t_{j-1} \mathrm{~d} t_{j+1} \cdots \mathrm{d} t_{n}\right)^{1 / s} \\
& =W_{j}^{1 / r}\left(\int_{\mathbb{R}_{+}^{n-1}} t_{1}^{\lambda_{1} c s} K\left(t_{1}, \cdots, t_{j-1}, 1, t_{j+1}, \cdots, t_{n}\right) \prod_{i \neq j}^{n} t_{i}^{-a_{i}} \mathrm{~d} t_{1} \cdots \mathrm{d} t_{j-1} \mathrm{~d} t_{j+1} \cdots \mathrm{d} t_{n}\right)^{1 / s} .
\end{aligned}
$$


Thereupon,

$$
W_{j} \geq \int_{\mathbb{R}_{+}^{n-1}} t_{1}^{\lambda_{1} c s} K\left(t_{1}, \cdots, t_{j-1}, 1, t_{j+1}, \cdots, t_{n}\right) \prod_{i \neq j}^{n} t_{i}^{-a_{i}} \mathrm{~d} t_{1} \cdots \mathrm{d} t_{j-1} \mathrm{~d} t_{j+1} \cdots \mathrm{d} t_{n} .
$$

If $\lambda_{1} c>0$, then $\lambda_{1} c s<0$ and

$$
\begin{aligned}
W_{j} & \geq \int_{0}^{\frac{1}{2}} \int_{\mathbb{R}_{+}^{n-2}} t_{1}^{\lambda_{1} c s} K\left(t_{1}, \cdots, t_{j-1}, 1, t_{j+1}, \cdots, t_{n}\right) \prod_{i \neq j}^{n} t_{i}^{-a_{i}} \mathrm{~d} t_{1} \cdots \mathrm{d} t_{j-1} \mathrm{~d} t_{j+1} \cdots \mathrm{d} t_{n} \\
& \geq\left(\frac{1}{2}\right)^{\lambda_{1} c s} \int_{0}^{\frac{1}{2}} \int_{\mathbb{R}_{+}^{n-2}} K\left(t_{1}, \cdots, t_{j-1}, 1, t_{j+1}, \cdots, t_{n}\right) \prod_{i \neq j}^{n} t_{i}^{-a_{i}} \mathrm{~d} t_{1} \cdots \mathrm{d} t_{j-1} \mathrm{~d} t_{j+1} \cdots \mathrm{d} t_{n} .
\end{aligned}
$$

Letting $s \rightarrow-\infty$, then $W_{j}=+\infty$. According to the convergence of $W_{j}$, we have a contradiction. Hence, $\lambda_{1} c>0$ is impossible.

If $\lambda_{1} c<0$, then $\lambda_{1} c s>0$ and

$$
\begin{aligned}
W_{j} & \geq \int_{2}^{+\infty} \int_{\mathbb{R}_{+}^{n-2}} t_{1}^{\lambda_{1} c s} K\left(t_{1}, \cdots, t_{j-1}, 1, t_{j+1}, \cdots, t_{n}\right) \prod_{i \neq j}^{n} t_{i}^{-a_{i}} \mathrm{~d} t_{1} \cdots \mathrm{d} t_{j-1} \mathrm{~d} t_{j+1} \cdots \mathrm{d} t_{n} \\
& \geq 2^{\lambda_{1} c s} \int_{2}^{+\infty} \int_{\mathbb{R}_{+}^{n-2}} K\left(t_{1}, \cdots, t_{j-1}, 1, t_{j+1}, \cdots, t_{n}\right) \prod_{i \neq j}^{n} t_{i}^{-a_{i}} \mathrm{~d} t_{1} \cdots \mathrm{d} t_{j-1} \mathrm{~d} t_{j+1} \cdots \mathrm{d} t_{n} .
\end{aligned}
$$

Letting $s \rightarrow-\infty$, then $W_{j}=+\infty$, which is contrary to the convergence of $W_{j}$; thus, $\lambda_{1} c<0$ cannot hold.

In conclusion, $\lambda_{1} c=0$; ; therefore, $c=0$, that is $\sum_{i=1}^{n} \frac{a_{i}}{\lambda_{i}}=\lambda+\sum_{i=1}^{n} \frac{1}{\lambda_{i}}$.

It has been proved that $(\mathrm{b}) \Rightarrow(\mathrm{c})$ and $(\mathrm{b}) \Rightarrow(\mathrm{a}),(\mathrm{a}) \Rightarrow(\mathrm{b})$ and $(\mathrm{c}) \Rightarrow(\mathrm{b})$, hence (a), (b) and (c) are equivalent to each other.

(iii) It can be obtained by the proof of (b) $\Rightarrow(a)$.

\section{Applications}

According to Theorem 1, the following several inequalities involving symmetry can be obtained.

Corollary 1. If $n \geq 2, \sum_{i=1}^{n} \frac{1}{p_{i}}=1\left(p_{i}>1\right), 0<\lambda_{i} \leq 1, \tilde{a}(i)=\left\{a_{m_{i}}(i)\right\} \in l_{p_{i}}^{p_{i}-1}(i=1,2$, $\cdots, n)$, then

$$
\begin{aligned}
& \sum_{m_{1}=1}^{\infty} \cdots \sum_{m_{n}=1}^{\infty} \frac{\min \left\{m_{1}^{\lambda_{1}}, \cdots, m_{n}^{\lambda_{n}}\right\}}{\max \left\{m_{1}^{\lambda_{1}}, \cdots, m_{n}^{\lambda_{n}}\right\}} \prod_{i=1}^{n} a_{m_{i}}(i) \\
\leq & n ! \prod_{i=1}^{n} \lambda_{i}^{\frac{1}{p_{i}}-1} \prod_{i=1}^{n}\|\tilde{a}(i)\|_{p_{i}, p_{i-1}},
\end{aligned}
$$

where the constant factor $n ! \prod_{i=1}^{n} \lambda_{i}^{\frac{1}{p_{i}}-1}$ is the best.

Proof. Let

$$
G\left(x_{1}^{\lambda_{1}}, \cdots, x_{n}^{\lambda_{n}}\right)=\frac{\min \left\{x_{1}^{\lambda_{1}}, x_{2}^{\lambda_{2}}, \cdots, x_{n}^{\lambda_{n}}\right\}}{\max \left\{x_{1}^{\lambda_{1}}, x_{2}^{\lambda_{2}}, \cdots, x_{n}^{\lambda_{n}}\right\}},
$$

then $G\left(u_{1}, u_{2}, \cdots, u_{n}\right)$ a is a homogeneous function of order $\lambda=0$.

Set $a_{i} p_{i}-1=p_{i}-1$, then $a_{i}=1$. Choose $a_{i}=1(i=1,2, \cdots, n)$ as the matching parameter, we have

$$
c=\sum_{i=1}^{n} \frac{p_{i}}{\lambda_{i}}-\left(\lambda+\sum_{i=1}^{n} \frac{1}{\lambda_{i}}\right)=\sum_{i=1}^{n} \frac{1}{\lambda_{i}}-\left(0+\sum_{i=1}^{n} \frac{1}{\lambda_{i}}\right)=0 .
$$


Hence $a_{i}=1(i=1,2, \cdots, n)$ is the best matching parameter.

Since $0<\lambda_{i} \leq 1(i=1,2, \cdots, n)$, then

$$
G\left(x_{1}^{\lambda_{1}}, \cdots, x_{i}^{\lambda_{i}}, \cdots, x_{n}^{\lambda_{n}}\right) x_{i}^{-a_{i}}=\frac{\min \left\{x_{1}^{\lambda_{1}}, \cdots, x_{i}^{\lambda_{i}}, \cdots, x_{n}^{\lambda_{n}}\right\}}{\max \left\{x_{1}^{\lambda_{1}}, \cdots, x_{i}^{\lambda_{i}}, \cdots, x_{n}^{\lambda_{n}}\right\}} x_{i}^{-1}
$$

decreases monotonically on $(0,+\infty)$ with respect to $x_{i}$. According to the results of [13], it is calculated that

$$
\begin{aligned}
W_{1} & =\int_{\mathbb{R}_{+}^{n-1}} \mathrm{G}\left(1, t_{2}^{\lambda_{2}}, \cdots, t_{n}^{\lambda_{n}}\right) \prod_{i=2}^{n} t_{i}^{-a_{i}} \mathrm{~d} t_{2} \cdots \mathrm{d} t_{n} \\
& =\int_{\mathbb{R}_{+}^{n-1}} \frac{\min \left\{1, t_{2}^{\lambda_{2}}, \cdots, t_{n}^{\lambda_{n}}\right\}}{\max \left\{1, t_{2}^{\lambda_{2}}, \cdots, t_{n}^{\lambda_{n}}\right\}} \prod_{i=2}^{n} t_{i}^{-1} \mathrm{~d} t_{2} \cdots \mathrm{d} t_{n} \\
& =\prod_{i=2}^{n} \frac{1}{\lambda_{i}} \int_{\mathbb{R}_{+}^{n-1}} \frac{\min \left\{1, u_{2}, \cdots, u_{n}\right\}}{\max \left\{1, u_{2}, \cdots, u_{n}\right\}} \prod_{i=2}^{n} u_{i}^{-1} \mathrm{~d} u_{2} \cdots \mathrm{d} u_{n} \\
& =n ! \prod_{i=2}^{n} \frac{1}{\lambda_{i}} .
\end{aligned}
$$

The conclusion of Corollary 1 can be obtained according to Theorem 1.

Corollary 2. If $n \geq 2, \sum_{i=1}^{n} \frac{1}{p_{i}}=1\left(p_{i}>1\right), 1 \leq k<n, \lambda>0, \lambda_{i}>0,0<a_{i}<1,0 \leq \sigma \leq$ $\min \left\{\frac{a_{1}}{\lambda 1}, \cdots, \frac{a_{k}}{\lambda k}\right\}, \tilde{a}(i)=\left\{a_{m_{i}}(i)\right\} \in l_{p_{i}}^{a_{i} p_{i}-1}(i=1,2, \cdots, n), \sum_{i=1}^{n} \frac{a_{i}}{\lambda_{i}}=\sigma-\lambda+\sum_{i=1}^{n} \frac{1}{\lambda_{i}}$, then

$$
\begin{aligned}
& \sum_{m_{1}=1}^{\infty} \cdots \sum_{m_{n}=1}^{\infty} \frac{\left(m_{1}^{\lambda_{1}}+\cdots+m_{k}^{\lambda_{k}}\right)^{\sigma}}{\left(m_{1}^{\lambda_{1}}+\cdots+m_{k}^{\lambda_{k}}+\cdots+m_{n}^{\lambda_{n}}\right)^{\lambda}} \prod_{i=1}^{n} a_{m_{i}}(i) \\
\leq & M_{0} \prod_{i=1}^{n}\|\tilde{a}(i)\|_{p_{i}, a_{i} p_{i-1}},
\end{aligned}
$$

where the constant factor

$$
M_{0}=\frac{1}{\Gamma(\lambda)} \prod_{i=1}^{n} \lambda_{i}^{\frac{1}{p_{i}}-1} \frac{\Gamma\left(\sigma+\sum_{i=1}^{k} \frac{1-a_{i}}{\lambda_{i}}\right)}{\Gamma\left(\sum_{i=1}^{k} \frac{1-a_{i}}{\lambda_{i}}\right)} \prod_{i=1}^{n} \Gamma\left(\frac{1-a_{i}}{\lambda_{i}}\right)
$$

is the best. 
Proof. Let

$$
G\left(x_{1}^{\lambda_{1}}, \cdots, x_{n}^{\lambda_{n}}\right)=\frac{\left(x_{1}^{\lambda_{1}}+\cdots+x_{k}^{\lambda_{k}}\right)^{\sigma}}{\left(x_{1}^{\lambda_{1}}+\cdots+x_{k}^{\lambda_{k}}+\cdots+x_{n}^{\lambda_{n}}\right)^{\lambda}},
$$

then $G\left(u_{1}, \cdots, u_{n}\right)$ is a homogeneous function of order $\sigma-\lambda$.

For $i>k$, notice that $\lambda>0, \lambda_{i}>0, a_{i}>0$, then

$$
G\left(x_{1}^{\lambda_{1}}, \cdots, x_{n}^{\lambda_{n}}\right) x_{i}^{-a_{i}}=\frac{\left(x_{1}^{\lambda_{1}}+\cdots+x_{k}^{\lambda_{k}}\right)^{\sigma}}{\left(x_{1}^{\lambda_{1}}+\cdots+x_{k}^{\lambda_{k}}+\cdots+x_{n}^{\lambda_{n}}\right)^{\lambda}} x_{i}^{-a_{i}}
$$

is monotonically decreasing on $(0,+\infty)$ with respect to $x_{i}$.

For $1 \leq i \leq k$, since $\lambda>0, \lambda_{i}>0, a_{i}>0,0 \leq \sigma \leq \min \left\{\frac{a_{1}}{\lambda 1}, \cdots, \frac{a_{k}}{\lambda k}\right\}$, then

$$
G\left(x_{1}^{\lambda_{1}}, \cdots, x_{n}^{\lambda_{n}}\right) x_{i}^{-a_{i}}=\frac{\left(x_{i}^{-a_{i} / \sigma} x_{1}^{\lambda_{1}}+\cdots x_{i}^{\lambda_{i}-a_{i} / \sigma}+\cdots+x_{i}^{-a_{i} / \sigma} x_{k}^{\lambda_{k}}\right)^{\sigma}}{\left(x_{1}^{\lambda_{1}}+\cdots+x_{i}^{\lambda_{i}}+\cdots+x_{n}^{\lambda_{n}}\right)^{\lambda}}
$$

is also monotonically decreasing on $(0,+\infty)$ with respect to $x_{i}$.

According to Lemma 2, we calculate

$$
\begin{aligned}
& W_{n}=\int_{\mathbb{R}_{+}^{n-1}} G\left(t_{1}^{\lambda_{1}}, \cdots, t_{n-1}^{\lambda_{n-1}}, 1\right) \prod_{i=1}^{n-1} t_{i}^{-a_{i}} \mathrm{~d} t_{1} \cdots \mathrm{d} t_{n-1} \\
& =\int_{\mathbb{R}_{+}^{n-1}} \frac{\left(t_{1}^{\lambda_{1}}+\cdots+t_{k}^{\lambda_{k}}\right)^{\sigma}}{\left(t_{1}^{\lambda_{1}}+\cdots+t_{k}^{\lambda_{k}}+t_{k+1}^{\lambda_{k+1}}+\cdots+t_{n-1}^{\lambda_{n-1}}+1\right)^{\lambda}} \prod_{i=1}^{n-1} t_{i}^{-a_{i}} \mathrm{~d} t_{2} \cdots \mathrm{d} t_{n-1} \\
& =\int_{\mathbb{R}_{+}^{n-k-1}} \prod_{i=k+1}^{n-1} t_{i}^{-a_{i}}\left(\int_{\mathbb{R}_{+}^{k}} \frac{\left(t_{1}^{\lambda_{1}}+\cdots+t_{k}^{\lambda_{k}}\right)^{\sigma}}{\left[\left(t_{1}^{\lambda_{1}}+\cdots+t_{k}^{\lambda_{k}}\right)+\left(t_{k+1}^{\lambda_{k+1}}+\cdots+t_{n-1}^{\lambda_{n-1}}\right)+1\right]^{\lambda}}\right. \\
& \left.\times \prod_{i=1}^{k} t_{i}^{-a_{i}} \mathrm{~d} t_{1} \cdots \mathrm{d} t_{k}\right) \mathrm{d} t_{k+1} \cdots \mathrm{d} t_{n-1} \\
& =\frac{\prod_{i=1}^{k} \Gamma\left(\frac{1-a_{i}}{\lambda_{i}}\right)}{\prod_{i=1}^{k} \lambda_{i} \Gamma\left(\sum_{i=1}^{k} \frac{1-a_{i}}{\lambda_{i}}\right)} \int_{\mathbb{R}_{+}^{n-k-1}} \prod_{i=k+1}^{n-1} t_{i}^{-a_{i}}\left(\int_{0}^{+\infty} \frac{u^{\sigma}}{\left[u+\left(t_{k+1}^{\lambda_{k+1}}+\cdots+t_{n-1}^{\lambda_{n-1}}\right)+1\right]^{\lambda}}\right. \\
& \left.\times u^{\sum_{i=1}^{k} \frac{1-a_{i}}{\lambda_{i}}-1} \mathrm{~d} u\right) \mathrm{d} t_{k+1} \cdots \mathrm{d} t_{n-1}
\end{aligned}
$$




$$
\begin{aligned}
& =\frac{\prod_{i=1}^{k} \Gamma\left(\frac{1-a_{i}}{\lambda_{i}}\right)}{\prod_{i=1}^{k} \lambda_{i} \Gamma\left(\sum_{i=1}^{k} \frac{1-a_{i}}{\lambda_{i}}\right)} \int_{0}^{+\infty} u^{\sigma+\sum_{i=1}^{k} \frac{1-a_{i}}{\lambda_{i}}-1}\left(\int_{\mathbb{R}_{+}^{n-k-1}} \frac{1}{\left[1+u+\left(t_{k+1}^{\lambda_{k+1}}+\cdots+t_{n-1}^{\lambda_{n-1}}\right)\right]^{\lambda}}\right. \\
& \left.\times \prod_{i=k+1}^{n-1} t_{i}^{-a_{i}} \mathrm{~d} t_{k+1} \cdots \mathrm{d} t_{n-1}\right) \mathrm{d} u \\
& =\frac{\prod_{i=1}^{k} \Gamma\left(\frac{1-a_{i}}{\lambda_{i}}\right)}{\prod_{i=1}^{k} \lambda_{i} \Gamma\left(\sum_{i=1}^{k} \frac{1-a_{i}}{\lambda_{i}}\right)} \frac{\prod_{i=1}^{n-1} \Gamma\left(\frac{1-a_{i}}{\lambda_{i}}\right)}{\prod_{i=k+1}^{n-1} \lambda_{i} \Gamma\left(\sum_{i=k+1}^{n-1} \frac{1-a_{i}}{\lambda_{i}}\right)} \int_{0}^{+\infty} u^{\sigma+\sum_{i=1}^{k} \frac{1-a_{i}}{\lambda_{i}}-1} \\
& \times\left(\int_{0}^{+\infty} \frac{1}{(1+u+v)^{\lambda}} v^{\sum_{i=k+1}^{n-1} \frac{1-a_{i}}{\lambda_{i}}-1} \mathrm{~d} v\right) \mathrm{d} u \\
& =\frac{\prod_{i=1}^{n-1} \Gamma\left(\frac{1-a_{i}}{\lambda_{i}}\right)}{\prod_{i=1}^{n-1} \lambda_{i} \Gamma\left(\sum_{i=1}^{k} \frac{1-a_{i}}{\lambda_{i}}\right) \Gamma\left(\sum_{i=k+1}^{n-1} \frac{1-a_{i}}{\lambda_{i}}\right)} \int_{\mathbb{R}_{+}^{2}} \frac{1}{(1+u+v)^{\lambda}} u^{\sigma+\sum_{i=1}^{k} \frac{1-a_{i}}{\lambda_{i}}-1} v^{\sum_{i=k+1}^{n-1} \frac{1-a_{i}}{\lambda_{i}}-1} \\
& \times \mathrm{d} u \mathrm{~d} v \\
& =\frac{\prod_{i=1}^{n-1} \Gamma\left(\frac{1-a_{i}}{\lambda_{i}}\right)}{\prod_{i=1}^{n-1} \lambda_{i} \Gamma\left(\sum_{i=1}^{k} \frac{1-a_{i}}{\lambda_{i}}\right) \Gamma\left(\sum_{i=k+1}^{n-1} \frac{1-a_{i}}{\lambda_{i}}\right)} \frac{\Gamma\left(\sigma+\sum_{i=1}^{k} \frac{1-a_{i}}{\lambda_{i}}\right) \Gamma\left(\sum_{i=k+1}^{n-1} \frac{1-a_{i}}{\lambda_{i}}\right)}{\Gamma\left(\sigma+\sum_{i=1}^{k} \frac{1-a_{i}}{\lambda_{i}}+\sum_{i=k+1}^{n-1} \frac{1-a_{i}}{\lambda_{i}}\right)} \\
& \times \int_{0}^{+\infty} \frac{1}{(1+t)^{\lambda}} t^{\sigma+\sum_{i=1}^{n-1} \frac{1-a_{i}}{\lambda_{i}}-1} \mathrm{~d} t \\
& =\frac{\prod_{i=1}^{n-1} \Gamma\left(\frac{1-a_{i}}{\lambda_{i}}\right) \Gamma\left(\sigma+\sum_{i=1}^{k} \frac{1-a_{i}}{\lambda_{i}}\right)}{\prod_{i=1}^{n-1} \lambda_{i} \Gamma\left(\sum_{i=1}^{k} \frac{1-a_{i}}{\lambda_{i}}\right) \Gamma\left(\sigma+\sum_{i=1}^{n-1} \frac{1-a_{i}}{\lambda_{i}}\right)} \frac{\Gamma\left(\sigma+\sum_{i=1}^{n-1} \frac{1-a_{i}}{\lambda_{i}}\right) \Gamma\left(\lambda-\sigma-\sum_{i=1}^{n-1} \frac{1-a_{i}}{\lambda_{i}}\right)}{\Gamma(\lambda)} \\
& =\frac{\prod_{i=1}^{n-1} \Gamma\left(\frac{1-a_{i}}{\lambda_{i}}\right) \Gamma\left(\sigma+\sum_{i=1}^{k} \frac{1-a_{i}}{\lambda_{i}}\right)}{\Gamma(\lambda) \prod_{i=1}^{n-1} \lambda_{i} \Gamma\left(\sum_{i=1}^{k} \frac{1-a_{i}}{\lambda_{i}}\right)} \Gamma\left(\frac{1-a_{n}}{\lambda_{n}}\right)=\frac{\prod_{i=1}^{n} \Gamma\left(\frac{1-a_{i}}{\lambda_{i}}\right) \Gamma\left(\sigma+\sum_{i=1}^{k} \frac{1-a_{i}}{\lambda_{i}}\right)}{\Gamma(\lambda) \prod_{i=1}^{n-1} \lambda_{i} \Gamma\left(\sum_{i=1}^{k} \frac{1-a_{i}}{\lambda_{i}}\right)} .
\end{aligned}
$$

Thereupon,

$$
W_{0}=\frac{1}{\lambda_{n}} W_{n}=\frac{1}{\Gamma(\lambda)} \prod_{i=1}^{n} \lambda_{i}^{-1} \frac{\Gamma\left(\sigma+\sum_{i=1}^{k} \frac{1-a_{i}}{\lambda_{i}}\right)}{\Gamma\left(\sum_{i=1}^{k} \frac{1-a_{i}}{\lambda_{i}}\right)} \prod_{i=1}^{n} \Gamma\left(\frac{1-a_{i}}{\lambda_{i}}\right) .
$$

It follows from Theorem 1 that Corollary 2 holds.

In Corollary 2, take $\sigma=0$; the following corollary can be obtained.

Corollary 3. Suppose that $n \geq 2, \sum_{i=1}^{n} \frac{1}{p_{i}}=1\left(p_{i}>1\right), \lambda>0, \lambda_{i}>0,0<a_{i}<1, \sum_{i=1}^{n} \frac{a_{i}}{\lambda_{i}}=$ $-\lambda+\sum_{i=1}^{n} \frac{1}{\lambda_{i}}, \tilde{a}(i)=\left\{a_{m_{i}}(i)\right\} \in l_{p_{i}}^{a_{i} p_{i}-1}(i=1,2, \cdots, n)$. Then

$$
\sum_{m_{1}=1}^{\infty} \cdots \sum_{m_{n}=1}^{\infty} \frac{1}{\left(m_{1}^{\lambda_{1}}+\cdots+m_{n}^{\lambda_{n}}\right)^{\lambda}} \prod_{i=1}^{n} a_{m_{i}}(i) \leq M_{0} \prod_{i=1}^{n}\|\tilde{a}(i)\|_{p_{i}, a_{i} p_{i}-1},
$$


where the constant factor

$$
M_{0}=\frac{1}{\Gamma(\lambda)} \prod_{i=1}^{n} \lambda_{i}^{\frac{1}{p_{i}}-1} \prod_{i=1}^{n} \Gamma\left(\frac{1-a_{i}}{\lambda_{i}}\right)
$$

is the best. result.

Take $a_{i}=\frac{\lambda_{i}}{p_{i}}(\sigma-\lambda)-1(i=1,2, \cdots, n)$ in Corollary 2, we can obtain the following Corollary 4. If $n \geq 2, \sum_{i=1}^{n} \frac{1}{p_{i}}=1\left(p_{i}>1\right), 1 \leq k<n, \lambda>0, \lambda_{i}>0,0<\lambda-\sigma<\frac{p_{i}}{\lambda i^{\prime}}$ $\alpha_{i}=\lambda_{i}(\sigma-\lambda)+p_{i}-1,0<\sigma<\left(1-\frac{1}{p_{i}}\right)^{-1}\left(\frac{1}{\lambda i}-\frac{\lambda}{p_{i}}\right), \tilde{a}(i)=\left\{a_{m_{i}}(i)\right\} \in l_{p_{i}}^{\alpha_{i}}(i=1,2, \cdots$, $n)$, then

$$
\begin{aligned}
& \sum_{m_{1}=1}^{\infty} \cdots \sum_{m_{n}=1}^{\infty} \frac{\left(m_{1}^{\lambda_{1}}+\cdots+m_{k}^{\lambda_{k}}\right)^{\sigma}}{\left(m_{1}^{\lambda_{1}}+\cdots+m_{k}^{\lambda_{k}}+\cdots+m_{n}^{\lambda_{n}}\right)^{\lambda}} \prod_{i=1}^{n} a_{m_{i}}(i) \\
\leq & M_{0} \prod_{i=1}^{n}\|\tilde{a}(i)\|_{p_{i}, \alpha_{i}},
\end{aligned}
$$

where the constant factor

$$
M_{0}=\frac{1}{\Gamma(\lambda)} \prod_{i=1}^{n} \lambda_{i}^{\frac{1}{p_{i}}-1} \frac{\Gamma\left(\sigma+\sum_{i=1}^{k} \frac{\lambda-\sigma}{p_{i}}\right)}{\Gamma\left(\sum_{i=1}^{k} \frac{\lambda-\sigma}{\lambda_{i}}\right)} \prod_{i=1}^{n} \Gamma\left(\frac{\lambda-\sigma}{\lambda_{i}}\right)
$$

is the best.

\section{Conclusions}

In this paper, based on some symmetric homogeneous kernels, the concept of the quasi-homogeneous kernel is proposed and extended to the high-dimensional case. Then, using the symmetry of the Hilbert-type inequality for each variable and the weight function method, the matching parameters $a_{1}, \cdots, a_{n}$ are introduced to obtain the Hilbert-type inequality of an $n$-multiple series. In Theorem 1 , the equivalent parameter conditions of the best constant factor of the $n$-multiple Hilbert-type inequality is established, and the parameter problem of constructing a Hilbert-type series inequality with the best constant factor is solved. As applications, some special cases are given in Corollaries 1-4, and many Hilbert series inequalities with symmetric quasi-homogeneous kernels and best constant factors are obtained.

Author Contributions: In the process of writing this article, the three authors discussed the relevant issues many times. Y.H. completed the draft of the full text, L.L. reviewed the full text and put forward suggestions, and finally B.H. revised and proofread it. All authors have read and agreed to the published version of the manuscript.

Funding: This work is supported by the NNSF of China (No. 12071491), Guangdong Basic and Applied Basic Research Foundation-Natural Science Foundation (No. 2021A1515010055) and Guangzhou Science and Technology Plan Project (No. 202102080177).

Institutional Review Board Statement: Not applicable.

Informed Consent Statement: Not applicable.

Data Availability Statement: Not applicable.

Acknowledgments: The authors are grateful to the reviewers for their valuable comments and suggestions to improve the quality of the manuscript. 
Conflicts of Interest: The authors declare no conflict of interest.

\section{References}

1. Weyl, H. Singuläre Integralgleichungen Mit Besonderer Berücksichtigung des Fourierschen Integraltheorems. Ph.D. Thesis, Universität Göttingen, Göttingen, Germany, 1908.

2. Hardy, G.H. Note on a theorem of Hilbert concerning series of positive terms. Proc. Lond. Math. Soc. 1925, 23, xlv-xlvi.

3. Krnić, M.; Pečarić, J. General of Hilbert's and Hardy's inequalities. Math. Ineq. Appl. 2005, 8, 29-51. [CrossRef]

4. Yang, B.C. On a new Hardy-hilbert's type inequality. Math. Ineq. Appl. 2004, 7, 355-363. [CrossRef]

5. Zhao, C.J.; Debnath, L. Some new type Hilbert integral inequalities. J. Math. Anal. Appl. 2001, 262, 411-418. [CrossRef]

6. Yang, B.C.; Wu, S.H.; Wang, A.Z. A new Hilbert-type inequality with positive homogeneous kernel and its equivalent forms. Symmetry 2020, 12, 342. [CrossRef]

7. Rassias, M.T.; Yang, B.C. On a Hilbert-type integral inequality in the whole plane related to the extended Riemann zeta function. Complex Anal. Oper. Theory 2019, 13, 1765-1782. [CrossRef]

8. Rassias, M.T.; Yang, B.C. On a Hilbert-type integral inequality related to the extended Hurwitz zeta function in the whole plane. Acta Appl. Math. 2019, 160, 67-80. [CrossRef]

9. Hong, Y.; Huang, Q.L.; Yang, B.C.; Liao, J. The necessary and sufficient conditions for the existence of a kind of Hilbert-type multiple integral inequality with the non-homogeneous kernel and its applications. J. Inequal. Appl. 2017, 2017, 316. [CrossRef]

10. Zhong, W.Y.; Yang, B.C. On multiple Hardy-Hilbert's integral inequality with kernel. J. Inequal. Appl. 2007, 2007, 1-17. [CrossRef]

11. Hong, Y.; He, B.; Yang, B.C. Necessary and sufficient conditions for the validity of Hilbert type integral inequalities with a class of quasi-homogeneous kernels and its application in operator theory. J. Math. Inequal. 2018, 12, 777-788. [CrossRef]

12. Fichtingoloz, G.M. A Course in Differential and Integral Calculus; People's Education Press: Beijing, China, 1957.

13. He, B.; Cao, J.F.; Yang, B.C. A brand new multiple Hilbert-type integral inequality. Acta Math. Sin. 2015, 58, 661-672. 